\title{
Effects of rest interval on isokinetic strength and functional performance after short term high intensity training
}

\author{
Danny M Pincivero, Scott M Lephart, Raj G Karunakara
}

\begin{abstract}
Objectives-The ability to maximally generate active muscle tension during resistance training has been established to be a primary determinant for strength development. The influence of intrasession rest intervals may have a profound effect on strength gains subsequent to short term high intensity training. The purpose of this study was to examine the effects of rest interval on strength and functional performance after four weeks of isokinetic training.
\end{abstract}

Methods-Fifteen healthy college aged individuals were randomly assigned to either a short rest interval group (group 1, $\mathbf{n}=8$ ) or a long rest interval group (group $2, n=7)$. Subjects were evaluated for quadriceps and hamstring isokinetic strength at 60 (five repetitions) and 180 (30 repetitions) degrees/second and functional performance with the single leg hop for distance test. One leg of each subject was randomly assigned to a four week, three days/week isokinetic strength training programme for concentric knee extension and flexion performed at 90 degrees/ second. Subjects in group 1 received a 40 second rest interval in between exercise sets, whereas subjects in group 2 received a 160 second rest period.

Results-A two factor analysis of variance for the pre-test-post-test gain scores (\%) showed significantly greater improvements for isokinetic hamstring total work and average power at 180 degrees/second for the trained limb of subjects in group 2 than their contralateral non-trained limb and the subjects in group 1 . Significantly greater improvements for the single leg hop for distance were also found for the trained limbs of subjects in both groups as compared with the non-trained limbs.

Conclusions-The findings indicate that a relatively longer intrasession rest period resulted in a greater improvement in hamstring muscle strength during short term high intensity training.

(Br F Sports Med 1997;31:229-234)

Keywords: fatigue; recovery; peak torque; quadriceps; hamstrings

Strength training programmes are commonly used to enhance performance, reduce the incidence of overuse injuries, and assist in the rehabilitation of orthopaedic and athletically induced musculoskeletal injuries. Optimally designed strength training programmes are based on sound scientific principles that ensure a progressive overload of the appropriate muscle or muscle groups. ${ }^{1}$ This is normally achieved by manipulating the volume and intensity of exercise on a consistent and systematic basis, which involves the athlete or patient performing one or more sets of a particular exercise interspersed with rest intervals. At the present time, much information on the optimal training load to be utilised during rehabilitation and strength training programmes is available. However, intrasession rest intervals (period of time between sets of exercise) is a component that has not been extensively studied. ${ }^{2}$

Adequate rest periods are necessary between bouts of strength exercise in order to off-set the detrimental effects of fatigue and to facilitate muscle recovery. ${ }^{34}$ However, it has also been proposed that the development of fatigue through the reduction or elimination of rest intervals may actually enhance strength development. ${ }^{56}$ It is also well documented that intense exercise induces peripheral muscle fatigue, which reduces the potential of skeletal muscles to exert active tension. ${ }^{78}$ The ability to restore neuromuscular activation, active muscle tension, and metabolic homoeostasis is a time dependent process, suggesting the importance of a non-contractile period of rest after exercise. ${ }^{9-11}$

Under conditions of high intensity fatiguing exercise, a vast number of mechanisms have been implicated as key factors contributing to decrements in muscle performance. Such mechanisms include the accumulation of $\mathrm{H}^{+}$ and monovalent inorganic phosphate $\left(\mathrm{H}_{2} \mathrm{PO}_{4}^{-}\right)$, decreases in phosphocreatine (PCr) as well as impairments in excitationcontraction coupling. ${ }^{711-16}$ The ability to restore these changes to pre-fatigue or resting conditions after high intensity exercise has been suggested to last from a few minute ${ }^{17-19}$ to over one hour. ${ }^{13}$ With respect to isokinetic torque, however, investigators have observed that quadriceps muscle performance appears to approach full recovery with two to three minutes of rest. ${ }^{20-22}$

The influence of varying intrasession rest intervals during the time course of strength training has been proposed to play a key role in strength development, ${ }^{2}$ although current evidence is limited and appears conflicting. ${ }^{6022}$ 
Since the ability to generate high active tensile forces in muscle is the primary stimulus for strength gains, the optimisation of rest periods in order to enhance subsequent bouts of muscular contractions seems prudent. ${ }^{2}$ It has been proposed, however, that metabolite accumulation during short term submaximal isometric quadriceps training may provide a significant stimulus for the development of strength. ${ }^{6}$ Although it has been established that neuromuscular adaptations, as well as localised muscle fibre structural and enzymic changes, occur as early as two to four weeks after high intensity strength training, ${ }^{23}{ }^{24}$ the short term effects of intrasession rest intervals remain to be adequately clarified.

Therefore the purpose of this study was to examine the influence of intrasession rest interval on isokinetic strength of the quadriceps and hamstrings and a functional performance task, the single leg hop for distance test, after four weeks of isokinetic strength training.

\section{Methods}

\section{SUBJECTS}

Subjects for this study consisted of 15 healthy college aged volunteers (mean (SD) age 21.7 (1.9) years, mean (SD) height $172.5(8.5) \mathrm{cm}$, mean (SD) weight $68.7(9.8) \mathrm{kg}$ ) with no previous history of injury to the lower extremity and no resistance training within the past six months. Before participation in this study, each subject provided written informed consent approved through the Biomedical Institutional Review Board at the University of Pittsburgh. Subjects were tested for functional lower extremity performance and isokinetic strength before and immediately after the four week training period.

\section{TESTING PROCEDURES}

Before testing, each subject completed a dynamic warm up period that consisted of cycling on a Fitron (Lumex Corp, Ronkonkoma, NY, USA) bicycle for five minutes at $60 \mathrm{rpm}$ followed by quadriceps and hamstring stretching. In order to reduce the possible effects of fatigue incurred during the isokinetic testing evaluation, each subject performed the single leg hop for distance test first. The order of limb testing for the hop and the isokinetic test was randomly selected.

\section{Functional performance assessment}

Functional lower extremity performance was assessed by a single leg hop for distance test. ${ }^{25-27}$ A tape measure marked in centimetres was placed across the floor in order to determine the distance jumped. Subjects were instructed to stand on one leg with toes positioned to the zero mark on the tape, with their arms by their sides. They were then instructed to hop forward as far as possible and to land on the same leg. In order to simulate a functional test, subjects were allowed to swing their arms forward as they jumped. In addition, they were instructed to wear running shoes to which they felt accustomed. The distance from the zero mark to their heel was recorded for three trials and then averaged. Each subject was given two to three practice trials before the test. In order to determine the relative distance jumped, the absolute average distance of the three trials was divided by the subject's leg length measured from the anterior superior iliac spine to the medial malleolus. This corrected relative value was then used for further statistical analysis.

\section{Isokinetic dynamometry}

Isokinetic strength was assessed with the Biodex System 2 Isokinetic Dynamometer (Biodex Medical Inc, Shirley, NY, USA). Subjects were placed in a comfortable upright seated position on the Biodex dynamometer chair and secured using thigh, pelvic, and torso straps to minimise extraneous body movements. The lateral femoral epicondyle was used as the bony landmark for matching the axis of rotation of the knee joint to the axis of rotation of the dynamometer resistance adaptor. Once the subject was placed in a position that allowed comfortable and unrestricted motion for knee extension and flexion from a position of 90 degrees of flexion to terminal extension, the following measurements were taken: seat height, seat inclination, dynamometer head height, and resistance pad level. These measures were recorded and stored in the Biodex Advantage Software program, version 4.0 (Biodex Medical Inc) to standardise the testing position for each individual subject. Gravity correction was obtained by measuring the torque exerted on the dynamometer resistance adaptor with the knee in a relaxed state at terminal extension. Values for the isokinetic variables measured were automatically adjusted for gravity by the Biodex Advantage Software program. Calibration of the Biodex dynamometer was performed according to the specifications outlined by the manufacturer's service manual. During the testing procedure, the cushion setting on the control panel for the ends of the range of motion were set to their lowest (hard) setting in order to reduce the effect of limb deceleration on the reciprocal motion. ${ }^{28}$

Reciprocal concentric isokinetic knee extension and flexion was assessed at two angular velocities: 60 and 180 degrees/second. Testing at each velocity started with five submaximal followed by two to three maximal repetitions for warm up purposes. Five maximal repetitions were then performed at 60 degrees/ second. Once both limbs had been tested, each subject was given a brief period of volitional recovery (about five minutes) and then asked to perform 30 maximal repetitions at 180 degrees/second. Subjects performed 30 repetitions in an attempt to assess the muscular endurance capability of the quadriceps and hamstrings. Values for peak torque (N.m), total work (N.m), and average power (W) were computed for the quadriceps and hamstrings at 60 and 180 degrees/second in addition to the work performed during the last one third of the 180 degrees/second test. Each subject performed the 60 degrees/second test before the 180 degrees/second test in an attempt to remove the effect of fatigue that may have occurred during the latter test velocity. During the testing procedure, subjects were required to 
fold their arms across their chest, and were given verbal encouragement as well as visual feedback from the Biodex monitor in an attempt to achieve maximal effort. ${ }^{29}{ }^{30}$ All testing procedures were conducted by the same investigator for all subjects. Improvements in isokinetic quadriceps and hamstring torque after training beyond the percentages outlined in table 1 have been accepted as clinically meaningful as these values represent standard errors of measurement for these variables. ${ }^{31}$

TRAINING PERIOD

Subjects were randomly assigned to one of two training groups. Subjects in group 1 received a short intrasession rest interval between exercise sets (40 seconds) corresponding to a $2: 1$ rest to work ratio and subjects in group 2 received a long intrasession rest interval between exercise sets (160 seconds) corresponding to an 8:1 rest to work ratio. Each subject trained one randomly assigned leg three days per week for four weeks on the Biodex System 2 Isokinetic Dynamometer. All subjects performed the identical training programme, with the exception of the intrasession rest interval. Subjects performed four sets of ten repetitions during each training session in the first week and increased by one set per session each week for the duration of the study. Maximal reciprocal concentric isokinetic knee extension and flexion was performed at a pre-set angular velocity of 90 degrees/second. This training velocity was selected as an intermediary between the two testing velocities in an attempt to stimulate strength adaptations at both velocities. Before each training session, subjects completed a dynamic warm up period and were positioned on the Biodex Isokinetic System in exactly the same way as during the testing procedure. During each training session, all subjects were provided visual feedback from the Biodex monitor and given verbal encouragement by the same investigator.

\section{STATISTICAL ANALYSIS}

The pre-test-post-test difference for peak torque, total work, average power, and the single leg hop for distance was analysed as a gain score (\%). This method of unconditional inference was used, as opposed to conditional inference through the analysis of covariance, in order to quantify clinically meaningful strength gains beyond the inherent variability of measurement associated with the Biodex isokinetic dynamometer. ${ }^{31} \mathrm{~A}$ two factor (group $x$

Table 1 Standard errors of measurement (\%) for concentric isokinetic strength of the quadriceps and hamstrings using the Biodex System II isokinetic dynamometer (data from Pincivero et al ${ }^{35}$ )

\begin{tabular}{llllll}
\hline & \multicolumn{2}{l}{60 degrees/second } & & \multicolumn{2}{l}{180 degrees/second } \\
\cline { 2 - 3 } \cline { 5 - 6 } \cline { 5 - 6 } & Quadriceps & Hamstrings & & Quadriceps & Hamstrings \\
\hline $\begin{array}{c}\text { Peak } \\
\text { torque }\end{array}$ & 4.8 & 4.9 & & 5.6 & 6.1 \\
$\begin{array}{c}\text { Total work } \\
\text { Average } \\
\text { power }\end{array}$ & 8.9 & 7.4 & & 9.6 & 7.4 \\
$\begin{array}{c}\text { Work last } \\
\text { third }\end{array}$ & 2.0 & 7.1 & & 9.5 & 7.6 \\
\hline
\end{tabular}

limb) analysis of variance was performed to detect significant main effects and interactions at a pre-set alpha level of $\mathrm{P}<0.05$.

\section{Results}

Tables 2 and 3 summarise the percentage changes in the isokinetic strength variables and the single leg hop for distance test. The results show significant main effects between the two groups for quadriceps average power at 60 degrees/second and quadriceps peak torque at 180 degrees/second, and the training group with the longer rest interval (group 2) showed greater improvements than group 1 (table 4). Significant main effects were also found between the trained and non-trained limbs for quadriceps average power at 60 degrees/second and the single leg hop for distance test, and the trained limb in both groups displayed statistically higher values (table 5 ).

Significant group $\mathrm{x}$ limb interactions were shown for the following: (1) hamstring peak torque at 60 degrees/second $\left(F_{1,13}=7.38, \mathrm{P}=\right.$ 0.018 ), (2) hamstring average power at 60 degrees/second $\left(F_{1,13}=4.67, P=0.05\right)$, (3) hamstring peak torque at 180 degrees/second $\left(F_{1,13}=7.27, \mathrm{P}=0.018\right)$, (4) hamstring total work at 180 degrees/second $\left(F_{1,13}=12.77, \mathrm{P}=\right.$ $0.0034)$, and (5) hamstring average power at 180 degrees/second $\left(F_{1,13}=12.48, P=0.0037\right)$. The results show that each limb in both groups experienced decrements for hamstring peak torque at 60 degrees/second; however, the non-trained limb of subjects in group 2 (long rest interval) showed greater decreases $(10.9 \%)$ than the trained contralateral limb and the non-trained limb of subjects in group 1. Similar findings were also evident for hamstring average power at 60 degrees/second, as the non-trained limb of subjects in group 2 experienced greater decreases $(11.1 \%)$ than the contralateral trained leg and the nontrained leg of subjects in group 1. The trained limb of subjects in group 2 experienced a positive change in hamstring peak torque at 180 degrees/second $(6.0 \%)$ as compared with the non-trained limb $(-9.3 \%)$ and with the subjects in group 1 who displayed reductions in strength that were greater than the corresponding standard error of measurement values presented in table 1. Large improvements for hamstring total work (fig 1) and average power (fig 2) at 180 degrees/second were shown by the trained limb of subjects in group 2 (17.5 and $20.2 \%$ respectively) while the contralateral non-trained leg and both legs of subjects in group 1 experienced minimal changes in strength.

\section{Discussion}

The major findings of the present study indicate that strength gain subsequent to maximal isokinetic training is affected by the manipulation of intrasession rest intervals. It appears that isokinetic hamstring muscle strength responds to a greater extent to isokinetic training and longer intrasession rest intervals than the quadriceps muscles. Moreover, functional performance also appeared to 
Table 2 Percentage change in isokinetic strength at 60 degrees/second after four weeks of training. Values are mean (SEM)

\begin{tabular}{lcllllr}
\hline & \multicolumn{2}{l}{ Group 1 $(n=8)$} & & \multicolumn{2}{l}{ Group 2 $(n=7)$} \\
\cline { 2 - 3 } \cline { 6 - 6 } \cline { 5 - 6 } & Trained & Non-trained & & Trained & Non-trained \\
\hline PT Quadriceps & $0.7(2.2)$ & $-0.1(3.5)$ & & $5.9(3.5)^{\star}$ & $2.5(3.1)$ \\
TW Quadriceps & $10.0(9.4)^{\star}$ & $11.8(6.5)^{\star}$ & & $4.5(4.3)$ & $2.3(4.3)$ \\
AP Quadriceps & $0.7(2.9)$ & $-2.6(3.6)^{\star}$ & & $8.1(3.0)^{\star}$ & $2.3(3.4)^{\star}$ \\
PT Hamstrings & $-9.5(2.7)$ & $-2.3(4.5)$ & & $-2.4(3.2)$ & $-10.9(3.4)^{\star}$ \\
TW Hamstrings & $-1.9(9.5)$ & $10.1(8.5)^{\star}$ & & $-3.7(2.3)$ & $-11.1(3.7)^{\star}$ \\
AP Hamstrings & $-6.1(4.4)$ & $-2.2(4.6)$ & & $2.9(3.8)$ & $-11.1(3.9)^{\star}$ \\
\hline
\end{tabular}

* Percentage change greater than standard error of measurement.

PT, peak torque; TW, total work; AP, average power.

Table 3 Percentage change in isokinetic strength at 180 degrees/second and the single leg hop for distance after four weeks of training. Values are mean (SEM)

\begin{tabular}{|c|c|c|c|c|}
\hline \multirow[b]{3}{*}{ PT Quadriceps } & \multicolumn{2}{|l|}{ Group $1(n=8)$} & \multicolumn{2}{|c|}{ Group $2(n=7)$} \\
\hline & Trained & Non-trained & \multirow{2}{*}{$\begin{array}{l}\text { Trained } \\
8.0(4.4)^{\star}\end{array}$} & \multirow{2}{*}{$\begin{array}{c}\text { Non-trained } \\
8.7(1.9)^{\star}\end{array}$} \\
\hline & $0.5 \quad(2.5)$ & $4.0 \quad(2.7)$ & & \\
\hline TW Quadriceps & 7.2 (4.7) & $12.5(4.8)^{\star}$ & $9.2(4.1)$ & $6.3(2.8)$ \\
\hline AP Quadriceps & $7.1 \quad 3.8)$ & $8.9(4.2)$ & $11.1(3.4)^{\star}$ & $5.4(3.0)$ \\
\hline WLT Quadriceps & $11.1(6.1)^{\star}$ & $11.1(6.4)^{\star}$ & $9.3(4.2)$ & $3.7(3.4)$ \\
\hline PT Hamstrings & $-8.3(5.9)^{\star}$ & $-7.4(5.0)^{\star}$ & $6.0(5.4)$ & $-9.3(5.7)$ \\
\hline TW Hamstrings & $-0.2 \quad(6.2)$ & $-1.0(6.4)$ & $17.5(7.1)^{\star}$ & $-7.0(3.8)$ \\
\hline AP Hamstrings & $0.02(6.8)$ & $-4.2 \quad(5.6)$ & $20.2(6.7)^{\star}$ & $-7.2(3.1)$ \\
\hline WLT Hamstrings & $6.6(7.1)$ & $-4.8 \quad(5.7)$ & $20.8(9.5)^{\star}$ & $-3.9(4.5)$ \\
\hline HOP & $4.5 \quad(2.1)$ & $0.02(1.7)$ & $1.3(1.5)$ & $-0.1(1.3)$ \\
\hline
\end{tabular}

$\star$ Percentage change greater than standard error of measurement.

PT, peak torque; TW, total work; AP, average power; WLT, work last third; HOP, single leg hop for distance.

Table 4 Significant main effects between the two training groups for quadriceps average power at 60 degrees/decond (AP60Q) and quadriceps peak torque at 180 degrees/second (PT180Q). Means and standard errors (SE) represent percent changes between pre-test and post-test evaluations

\begin{tabular}{lllllll}
\hline & Group & $n$ & Mean & $S E$ & F value & P value \\
\hline AP60Q & 1 & 8 & -0.96 & 2.3 & 10.33 & 0.007 \\
& 2 & 7 & 5.2 & 2.3 & & \\
PT180Q & 1 & 8 & 2.3 & 1.83 & 6.75 & 0.02 \\
& 2 & 7 & 8.4 & 2.3 & & \\
\hline
\end{tabular}

Table 5 Significant main effects between the trained and non-trained limbs for quadriceps average power at 60 degrees/second ( $A P 60 Q)$, hamstring work performed in the last one third at 180 degrees/second (WLTH) and the single leg hop for distance(HOP). Mean and standard errors (SE) represent percentage changes between pre-test and post-test evaluations

\begin{tabular}{lllclll}
\hline & Limb & $n$ & Mean & $S E$ & F value & P value \\
\hline AP60Q & Trained & 15 & 4.66 & 2.3 & 6.02 & 0.03 \\
& Non-trained & 15 & -0.03 & 2.5 & & \\
WLTH & Trained & 15 & 14.2 & 6.13 & 10.31 & 0.007 \\
& Non-trained & 15 & -4.4 & 3.5 & & \\
HOP & Trained & 15 & 2.81 & 1.3 & 5.70 & 0.03 \\
& Non-trained & 15 & -0.06 & 0.99 & & \\
& & & & & &
\end{tabular}

be improved after training, although it was not affected by intrasession rest interval manipulation.

INTRASESSION REST INTERVAL

The results indicate that the manipulation of intrasession rest intervals had a significant effect on isokinetic strength with respect to hamstring peak torque and average power at 60 degrees/second and hamstring peak torque, total work, and average power at 180 degrees/ second. However, hamstring total work and average power at 180 degrees/second for the trained limb of subjects in group 2 were the only variables that showed increases beyond their respective standard error of measurement values (17.5 and $20.2 \%$ respectively). This finding suggests that sustained mechanical

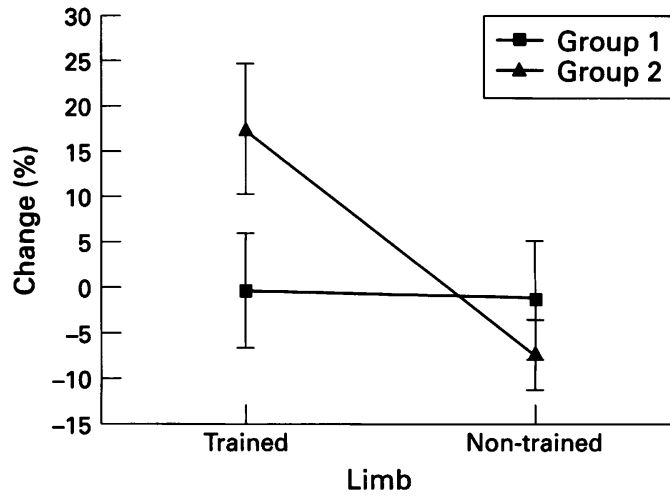

Figure 1 Significant group $\times$ limb interaction for percentage changes in hamstring total work at 180 degrees/second.

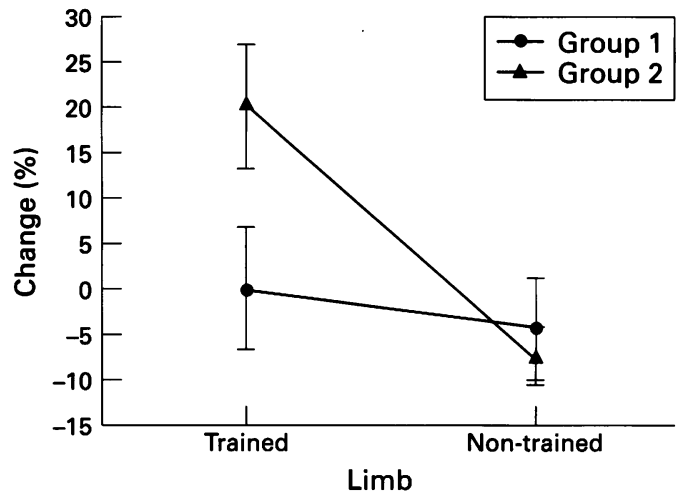

Figure 2 Significant group $\times$ limb interaction for percentage changes in hamstring average power at 180 degrees/second.

work performed by the hamstring muscles over time (30 s) as well as per unit of time is positively affected by incorporating intrasession rest intervals of 160 seconds during a short term strength training period.

Adequate recovery of muscle force within a training session is a necessary prerequisite for the generation of tension in subsequent contractions. Such benefits of utilising a relatively longer intrasession rest interval during strength training is that sufficient time is allowed for the involved muscle to replenish intramuscular stores of ATP and PCr. ${ }^{17-19}$ To examine the effects of rest interval on muscle tension development, Edman and Lou ${ }^{10}$ applied a one second fused tetanic electrical stimulus to the anterior tibialis of the frog, Rana temporarium, in which a greater reduction in force was observed when the rest intervals were progressively reduced from 300 to 15 to 1 second. The 15 second rest interval resulted in a $20-30 \%$ reduction in tetanic force, whereas the one second rest interval produced a $50-60 \%$ decrease compared with the control value. ${ }^{10}$

Bilcheck et $a l^{20}$ found that a 2.5 minute rest interval was sufficient to allow the recovery of isokinetic quadriceps muscle strength after three sets of 30 repetitions performed at 120 degrees/second. Similarly, Touey et $a l^{2}$ observed that an intrasession rest interval of one minute allowed adequate recovery of the quadriceps muscles after multiple sets of isokinetic exercise performed at 180 degrees/second. However, it was also shown that a rest interval 
of $120 \mathrm{~s}$ was necessary for optimal muscle recovery. ${ }^{22}$

It has been suggested by Rooney et alp that processes associated with muscle fatigue contribute to the strength training stimulus, as it was shown that the elimination of rest periods produced greater strength gains after six weeks of isotonic biceps brachii training than sessions utilising rest periods. However, the design of this study was such that one set of either six or ten repetitions was performed during each training session. A possible explanation for these results may be that an insufficient volume of mechanical work was demanded of the working muscle to produce any significant strength gains. Similar conclusions were derived by Schott et $a l,{ }^{6}$ who trained seven subjects three days per week for 14 weeks with a quadriceps isometric protocol equivalent to $70 \%$ of their maximum vital capacity. Subjects who performed sustained contractions for 30 seconds with a two minute intrasession rest interval, as compared with subjects performing intermittent three second contractions with a one minute rest period, produced greater strength gains (median $54.7 \%$ v 31.5\%) Schott et al suggested that metabolic changes in the muscle, as characterised by greater reductions in $\mathrm{pH}$ and $\mathrm{PCr}$, are involved in the adaptational response to strength training Contrary to these findings, Robinson $e t a p^{2}$ found a significantly greater increase in one repetition maximum squat strength after five weeks of training in subjects utilising a three minute rest interval as compared with subjects using a 30 second rest period. The results from the present investigation appear to concur with the results obtained by Robinson et al, ${ }^{32}$ as isokinetic hamstring strength improved significantly more when an intrasession rest interval of 160 seconds rather than 40 seconds was utilised.

\section{FUNCTIONAL PERFORMANCE}

The results from the present study indicate that functional performance is not affected by rest interval manipulation. Although a significantly greater improvement in the single leg hop for distance test was found for the trained limbs in both groups, concentric isokinetic torque and functional performance have yielded some interesting findings within the literature. Robertson and Fleming ${ }^{33}$ showed that the muscles surrounding the knee joint contributed only $3.9 \%$ to the generation of energy during a standing long jump. Furthermore, extensor moments observed at the knee, ankle, and hip showed that the knee joint primarily acts as an energy absorber, whereas the hip and ankle provide most of the mechanical energy for propulsion. It has recently been established that the relation between isokinetic hamstring total work and average power at 180 degrees/second correlates statistically more highly with the single leg hop for distance than the same variables for the quadriceps. ${ }^{34}$ The greater improvements for these two isokinetic variables after training, as detected by the statistically significant interactions, may suggest that the improvement in hamstring strength may be a mediating factor for the enhancement of functional performance.

\section{CROSS LIMB TRANSFER}

It has been well established that strength improvements after short term training are mediated largely by neurological factors. ${ }^{123}$ It therefore has been proposed that an increased outflow of impulses from the motor cortex of the brain has similar effects with respect to improvements in motor unit recruitment in both the trained and non-trained limbs. ${ }^{35}$

Previous studies have presented conflicting evidence with respect to the cross limb transfer effect after unilateral strength training. Krotkiewski $e t a l^{\beta 6}$ and Komi et $a l^{37}$ showed that single leg isokinetic and isometric training for the quadriceps respectively resulted in a strength improvement of the non-trained limb that ranged from 4 to $11 \%$. However, Tesch and Karlsson, ${ }^{38}$ Young et $a l^{39}$ and Jones and Rutherford ${ }^{40}$ did not find any strength improvements of the untrained quadriceps muscles after short term (5-12 weeks) unilateral strength training. Kannus et $a l^{\beta 5}$ found that after 7 weeks of combined unilateral isometric and isokinetic training of the quadriceps and hamstring muscles, isokinetic strength improvements in the non-trained leg ranged from 5 to $11 \%$. The results obtained by Kannus et $a l^{\beta 5}$ appear to be similar to those of the present study as strength increases in the non-trained limb ranged from 2.3 to $11.8 \%$ at 60 degrees/second and 8.7 to $12.5 \%$ at 180 degrees/second. Kannus et $a l^{\beta 5}$ suggested that previous studies lacked documentation on statistical power calculations to estimate the probability of accepting a null hypothesis - that is, no cross limb transfer effect. However, the results from the present investigation suggest that the reliability of the strength testing instrument may play a critical role in the interpretation of the results. The precision of measurement has been estimated for the Biodex dynamometer, ${ }^{31}$ as indicated by the standard error of measurement values in table 1 , whereas instrumentation error did not appear to be adequately addressed in previous studies. As a result of this presumption, clinically meaningful strength changes after training can be more appropriately interpreted if measurement variability is known. Otherwise, true strength improvements that range from 5 to $11 \%$ may be difficult to distinguish from test-retest error. In addition, the most significant limitation of the present investigation is the use of the non-trained limb as an internal control. Neurological adaptations have been documented as the primary mechanism for strength gains during the early time course of training which may manifest cross limb transfer effects. However, it is suggested that instrumentation reliability and precision is a critical factor for monitoring training induced strength changes beyond the inherent variability of the strength testing device. In addition, it should be noted that the training velocity of 90 degrees/second was chosen as an intermediate between the testing velocities of 60 and 180 
degrees/second. Although training induced changes in strength were observed at both 60 and 180 degrees/second, the issue of isokinetic velocity specificity remains beyond the scope of the present study.

\section{Conclusions}

The present investigation has shown that a relatively longer intrasession rest interval allows a greater enhancement of hamstring muscle strength than a corresponding shorter rest period. It was also evident that isokinetic quadriceps torque improved after training, as did functional performance. These improvements, however, do not appear to be affected by rest interval manipulation.

1 Kraemer WJ, Fleck SJ, Evans WJ. Strength and power training: physiological mechanism of adaptation. Exerc Sport $S_{c i}$ ing: physiological me

2 Weiss LW. The obtuse nature of muscular strength: the conribution of rest to its development and expression. Fournal of Applied Sport Science Research 1991;5:219-27.

3 Fry AC, Kraemer WJ, Van Borselen F, Lynch JM, Marsit JL, Roy EP, et al. Performance decrements with high-intensity resistance exercise overtraining. Med Sci Sports Exerc 1994; 26:1165-73

4 Kuipers H, Keizer HA. Overtraining in elite athletes: review and directions for the future. Sports Med 1988;6:79-92.

5 Rooney KJ, Herbert RD, Balnave RJ. Fatigue contributes to the strength training stimulus. Med Sci Sports Exerc 1994;26:1160-4.

6 Schott J, McCully K, Rutherford OM. The role of metabolites in strength training. II. Short versus long isometric lites in strength training. II. Short versus long

7 Allen DG, Lannergren J, Westerblad H. Muscle cell function during prolonged activity: cellular mechanisms of function during prolonged activity: cell

8 Williams JH, Klug GA. Calcium exchange hypothesis of Williams JH, Klug GA. Calcium exchange hypothesis of 1995;18:421-34.

9 Baker AJ, Kustov KG, Miller RG, Weiner MW. Slow force recovery after long-duration exercise: metabolism and activation factors in muscle fatigue. $\mathcal{F}$ Appl Physiol 1993;74 2294-300.

10 Edman KAP, Lou F. Myofibrillar fatigue versus failure of activation during repetitive stimulation of frog muscle fibres. F Physiol 1992;457:655-73.

11 Miller RG, Giannini D, Milner-Brown HS, Layzer RB, Korestsky AP, Hooper D, Weiner MW. Effects of fatiguing exercise on high-energy phosphates, force, and EMG: evidence for three phases of recovery. Muscle Nerve 1987;10:810-21.

12 Boska MD, Moussavi RS, Carson PJ, Weiner MW, Mille RG. The metabolic basis of recovery after fatiguing exercise RG. The metabolic basis of recovery after fati
of human muscle. Neurology $1990 ; 40: 240-4$.

13 Moussavi RS, Carson PJ, Boska MD, Weiner MW, Miller RG. Nonmetabolic fatigue in exercising human muscle. Neurology 1989;39:1222-6.

14 Bendahan D, Jammes Y, Salvan AM, Badier M, ConfortGouny S, Guuillot C, Cozzone PJ. Combined electromyography- ${ }^{31} \mathrm{P}$-magnetic resonance spectroscopy study of human muscle fatigue during static contraction. Muscle Nerve 1996;19:715-21.

15 Cairns SP, Dulhunty AF. High-frequency fatigue in rat skeletal muscle: role of extracellular ion concentrations. Muscle Nerve 1995;18:890-8.

16 Lindinger I, McKelvie RS, Heigenhauser GJF. $\mathrm{K}^{+}$and Lacdistribution in humans during and after high-intensity exercise: role in muscle fatigue attenuation? $\mathcal{F} A$ ppl Physiol 1995;78:765-77.

17 Hultman E, Bergstrom J, McLennan, Anderson N. Breakdown and resynthesis of phosphorylcreatine and adenosine down and resynthesis of phosphorylcreatine and adenosine Scand $f$ Clin Lab Invest 1967;19:56-66.
18 Takahashi H, Mitsuharu I, Fujimoto K, Katsuta S, Anno I, Niitsu M, Itai Y. Control of the rate of phosphocreatine resynthesis after exercise in trained and untrained human resynthesis after exercise in trained and untrained human

19 Tesch PA, Wright JE. Recovery from short term intense exercise: its relation to capillary supply and blood lactate concentration. Eur F Appl Physiol 1983;52:98-103.

20 Bilcheck HM, Kraemer WJ, Maresh CM, Zito MA. The effects of isokinetic fatigue on recovery of maximal isokinetic concentric and eccentric strength in women. fournal of Strength and Conditioning Research 1993;7:43-50.

21 Hakkinen K, Komi PV. Effects of fatigue and recovery on electromyographic and isometric force- and relaxationtime characteristics of human skeletal muscle. Eur $¥$ Appl Physiol 1986;55:588-96.

22 Touey PR, Sforzo GA, McManis BG. Effect of manipulating rest periods on isokinetic muscle performance. Med Sci Sports Exerc 1994;26:S170.

23 Moritani T, DeVries HA. Neural factors versus hypertrophy in the time course of muscle strength gain. Am $\mathcal{F}$ Phys Med Rehabil 1979;58:115-30.

24 Staron RS, Karapondo DL, Kraemer WJ, Fry AC, Gordon $\mathrm{SE}$, Falkel JE, et al. Skeletal muscle adaptations during early phase of heavy-resistance training in men and women. f Appl Physiol 1994;76:1247-55.

25 Booher LD, Hench KM, Worrell TW, Stikeleather J. Reliability of three single-leg hop tests. Fournal of Spor Rehabilitation 1993;2:165-70.

26 Daniel DM, Stone ML, Riehl B, et al. A measurement of lower limb function: the one leg hop for distance. Am $\mathcal{F}$ Knee Surg 1988;1:212-24.

27 Wiklander J, Lysholm J. Simple tests for surveying muscle strength and muscle stiffness in sportsmen. Int $\mathcal{F}$ Sports Med 1987;8:50-4.

28 Taylor NAS, Sanders RH, Howick EI, Stanley SN. Static and dynamic assessment of the Biodex dynamometer. Eur $f$ Appl Physiol 1991;62:180-8.

29 Hald RD, Bottien EJ. Effect of visual feedback on maxima and submaximal isokinetic test measurements of normal andriceps and hamstrings. $f$ Orthop Sports Phys Ther 1987;9:86-93.

30 McNair PJ, Depledge J, Brettklelly M, Stanley SN. Verbal encouragement: effects of maximum effort voluntary mus cle activation. Br F Sport Med 1996;30:243-5.

31 Pincivero DM, Lephart SM, Karunakara RG. Reliability and precision of isokinetic strength and muscular endurance for the quadriceps and hamstrings. Int $f$ Sport Med 1996;18:115-19.

32 Robinson JM, Stone MH, Johnson RL, Penland CM, Warren BJ, Lewis RD. Effects of different weight training exercise/rest intervals on strength, power and high intensity exercise endurance. Fournal of Strength and Conditioning Research 1995;9:216-21.

33 Robertson DGE, Fleming D. Kinetics of standing broad and vertical jumping. Can $\mathcal{f}$ Sport Sci 1987;12:19-23.

34 Pincivero DM, Lephart SM, Karunakara RG. The relation between open and closed kinematic chain assessment of knee strength and functional performance. Clinical fournal of Sports Medicine 1997;7:11-16.

35 Kannus P, Alosa D, Cook L, Johnson RJ, Renström P, Pope $M$, et al. Effect of one-legged exercise on the strength, power and endurance of the contralateral leg. Eur $\mathcal{F}$ App Physiol 1992;64:117-26.

36 Krotkiewski M, Aniansson A, Grimby G, Bjöntorp P, Sjöström $L$. The effect of unilateral isokinetic strength training on local adipose and muscle tissue morphology, training on local adipose and muscle tissue morphology,
thickness, and enzymes. Eur $¥$ Appl Physiol 1979;42:27181 .

37 Komi PV, Viitasalo JT, Rauramaa R, Vihko V. Effect of isometric strength training on mechanical, electrical, and metabolic aspects of muscle function. Eur $\mathcal{f}$ Appl Physiol 1978;40:45-55.

38 Tesch PA, Karlsson J. Effects of exhaustive, isometric training on lactate accumulation in different muscle fiber types. Int $\mathcal{f}$ Sports Med 1984;5:89-91.

39 Young A, Stokes M, Round JM, Edwards RHT. The effect of high-resistance training on the strength and crosssectional area of the human quadriceps. Eur 7 Clin Invest 1983;13:411-17.

40 Jones DA, Rutherford OM. Human muscle strength training: the effects of three different regimes and the nature of the resultant changes. $\mathcal{F}$ Physiol 1987;391:1-11. 